\title{
Breakup locations: Intertwining effects of nuclear structure and reaction dynamics
}

\author{
M. Dasgupta*, E. C. Simpson, D. H. Luong, Sunil Kalkal, \\ K. J. Cook, I. P. Carter, D. J. Hinde, and E. Williams
}

Department of Nuclear Physics, Research School of Physics and Engineering, Australian National University, ACT 2601, Australia

\begin{abstract}
Studies at the Australian National University aim to distinguish breakup of the projectile like-nucleus that occurs when approaching the target from that when receding from the target. Helped by breakup simulations, observables have been found that are sensitive to the breakup location, and thus to the mean-lives of unbound states; sensitivity to even sub-zeptosecond lifetime is found. These results provide insights to understand the reaction dynamics of weakly bound nuclei at near barrier energies.
\end{abstract}

Understanding the interactions of weakly bound nuclei, and their reaction outcomes, is a key challenge in nuclear reactions research [1]. For well-bound nuclei, collisions near the barrier are reasonably well-described by including couplings to quantum states of the approaching nuclei. For weakly bound nuclei, the reaction dynamics has added complexity due the presence of low-lying particle unbound states. The situation is made even more challenging by the fact that weakly bound nuclei can breakup not only following direct excitation of continuum states (direct breakup), but also following nucleon transfer. The latter occurs when the neighbouring nuclei, formed by transfer, are themselves weakly bound or unbound. Modelling this is a major theoretical challenge, and currently there is no quantum

*E-mail: mahananda.dasgupta@anu.edu.au 
model that describes all breakup modes and their effect on other reaction channels simultaneously.

One of the first unambiguous experimental demonstrations of the effects of weak binding on other reaction channels came from using the concept of fusion barrier distributions [2]. Using the experimentally determined average barrier energy, it was shown that above-barrier complete fusion cross-sections of the weakly-bound nucleus ${ }^{9} \mathrm{Be}+{ }^{208} \mathrm{~Pb}$ is suppressed [2] compared with the expectations of well bound nuclei. Complete fusion suppression has since been seen in many reactions with weakly bound stable nuclei [3-9]. However, studies on medium-mass and light target nuclei are sparse as experimentally it is difficult to separate complete fusion (experimentally defined as capture of the full charge of the projectile) from incomplete fusion (only part of the projectile charge is captured). In the case of radioactive nuclei too, the majority of experimental observations agree that suppression is found above the barrier [10].

It was natural to think that the above-barrier suppression was due to breakup of weakly bound nuclei reducing the flux of intact nuclei at the barrier radius. Indeed, the relationship [8] between observed suppression, quantified by $1-F_{C F}$ with $F_{C F}$ being the fraction of complete fusion suppression, and the energy required for direct breakup, shown in Fig. 1, seemed to support this idea. However, many works (e.g. [11-14]) have indicated that the picture is not so simple, as transfer-triggered breakup, which can be important, is neglected. In ${ }^{6} \mathrm{Li},{ }^{7} \mathrm{Li}$ and ${ }^{9} \mathrm{Be}$ induced reactions for example, these channels are highly probable. This is demonstrated in Fig. 1(b), which shows coincident charged particles that are detected following collisions of ${ }^{7} \mathrm{Li}$ with ${ }^{208} \mathrm{~Pb}$. Only events following breakup close to the target are shown due to their relevance to fusion, as will be discussed later. It is clear from Fig. 1(b) that apart from $\alpha-t$ pairs from direct breakup of ${ }^{7} \mathrm{Li}, \alpha-d$, and $\alpha-p$ and $\alpha-\alpha$ coincidences, arising from breakup following stripping of $n, 2 n$ and pickup of $p$ respectively, are significant contributors $[13,14]$. Therefore, in this manuscript, the term breakup will include both direct and transfer-triggered modes.

In the absence of a quantum model, a classical approach [15] was developed to relate measured probabilities for various breakup modes at below barrier energies to above barrier fusion suppression. The classical model requires knowledge of probability of breakup in the incoming trajectory as a function of centre-to-centre separation of the two colliding nuclei. This information has been obtained by making measurements at a range of below barrier energies, where fragment absorption is minimal and thus interpretation of breakup is unambiguous $[13,14,16]$. This gives an exponential 
(a)

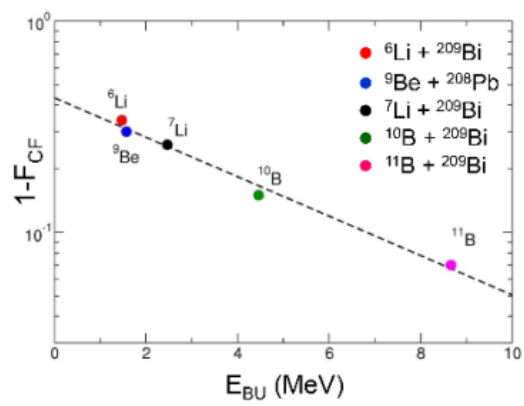

(b)

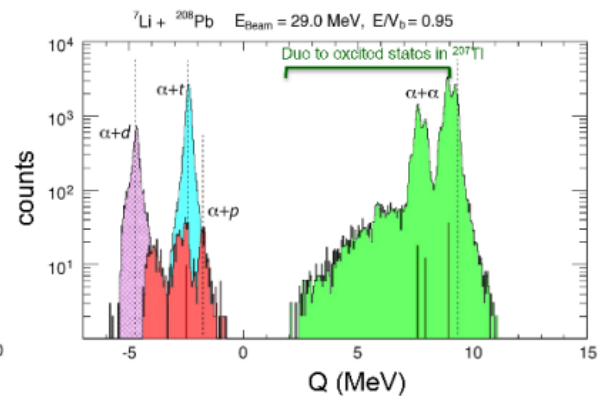

Figure 1: (Color online) (a) The experimentally determined suppression of complete fusion $[2,5,8]$ as a function of the energy required for direct breakup of the projectile for the reactions indicated. The quantity $F_{C F}$ is the observed fraction of complete fusion. Whilst this strong correlation has led to the notion that complete fusion suppression is linked to direct breakup, it masks the fact that a large proportion of breakup occurs via nucleon-transfer as shown in panel (b) for near-target breakup events (see text) that are observed following interactions of ${ }^{7} \mathrm{Li}$ with ${ }^{208} \mathrm{~Pb}[13,14]$.

fall-off with radial separation, and allows extrapolation to above-barrier energies. The investigations of breakup that followed focussed attention on the position at which breakup occurs due to its importance to fusion suppression. Complete fusion is expected to be suppressed if breakup of the projectile-like nucleus occurs whilst approaching the target, before reaching the fusion barrier radius. However, complete fusion will hardly be affected if the projectile-like nucleus passes inside the fusion barrier before disintegrating. This paper discusses recent work at the Australian National University aimed at distinguishing these two scenarios.

\section{Identifying breakup modes}

The experiments were performed using pulsed beams from the Heavy Ion Accelerator Facility at the Australian National University. Recent studies have focussed on a range of targets to study the dependence of the breakup modes on target mass $(A)$ and charge $(Z)$. The charged breakup fragments were detected in coincidence using an array of four double sided Silicon strip detectors with a large coverage in both scattering angle $\theta$ and azimuthal angle $\phi$. The signals in intersecting arcs and sectors provide information on $(\theta, \phi)$ of the detected particles. This information for each of the coincident fragments, along with their measured energy allows kinematic reconstruction of events. From the measured energies, angles and known beam energy, the 
Q-value for each event can be calculated. The Q-value spectrum [14] for events that arise from near-target breakup in collisions of ${ }^{7} \mathrm{Li}$ with ${ }^{208} \mathrm{~Pb}$ is shown in Fig. 1(b). It is clear that in addition to direct breakup into $\alpha$ and $t$ clusters, breakup via other modes is significant [14]. The transferinduced breakup modes arise as the nuclei that are populated following nucleon transfer are themselves weakly bound or unstable e.g. ${ }^{8} \mathrm{Be}$ formed following proton pickup breaks up into $\alpha-\alpha$, and ${ }^{5} \mathrm{Li}$ formed following $2 n$ stripping breaks into $\alpha-p$. These experiments, and those that followed using lighter targets, have all shown the dominance of transfer triggered breakup. The component of direct breakup is negligible for interactions with $\mathrm{Ni}$ and lower $Z$ targets. However, as discussed in the introduction, knowing the breakup modes alone is not sufficient to understand fusion suppression. A knowledge of the breakup location is crucially important.

\section{Relative energies of fragments as an indicator of breakup locations}

The relative energy of two coincident breakup fragments has been used in recent works $[13,14,16]$ to get information on the location of breakup. The concept is discussed here using the example of ${ }^{8} \mathrm{Be}$, which is populated in reactions involving ${ }^{7} \mathrm{Li}$ following $p$ pickup or in ${ }^{9} \mathrm{Be}$ induced reactions following $n$-stripping. The ground state of ${ }^{8} \mathrm{Be}$ is unstable against $\alpha$-decay by $92 \mathrm{keV}$ and has a mean-life of $10^{-16} \mathrm{~s}$. The mean-life is very long compared with the typical reaction time scale of $10^{-21} \mathrm{~s}$. The first excited state of ${ }^{8} \mathrm{Be}$ is much shorter lived, with a mean-life close to the reaction time scale. If ${ }^{8} \mathrm{Be}$ is populated in its ground state then, at the sub-barrier energies at which the measurements are made, it will recede far from the target before it breaks into two $\alpha$-particles. The relative energy of the two breakup $\alpha$-particles is therefore expected to be equal to $92 \mathrm{keV}$. If however ${ }^{8} \mathrm{Be}$ is populated in its excited state, its breakup can occur soon after its formation, while it is still in close proximity to the target. The Coulomb interaction between the target and the fragments will alter their relative velocity and thus the relative energy. The experimentally measured relative energy spectra [14], shown in Fig. 2, follows this expectation: at low energy there is a narrow peak which arises from breakup of the ${ }^{8} \mathrm{Be}$ ground state. The higher relative energy events arise due to breakup from the second resonant state of ${ }^{8} \mathrm{Be}$ and higher excitations. This realization means that a distinction between breakup near the target and far from the target (asymptotic) can be made experimentally. It is also clear that for fusion suppression, our focus must be 


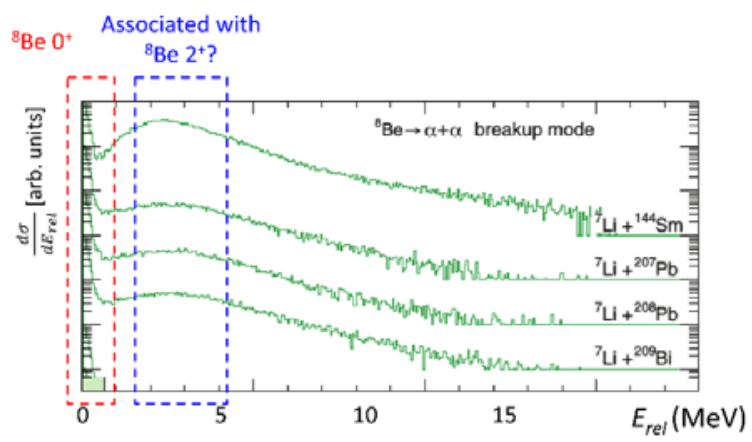

Figure 2: (Color online) Relative energy spectra for the two coincident $\alpha$-particles from the breakup of ${ }^{8} \mathrm{Be}$ formed via $p$-pickup by ${ }^{7} \mathrm{Li}$ incident on various targets. The narrow peak at relative energy of $92 \mathrm{keV}$ is due to breakup of ${ }^{8}$ Be ground state. The higher relative energy components arise due to breakup of excited states of ${ }^{8} \mathrm{Be}$ (see text).

on near target breakup processes (called prompt breakup in Refs. [13,14,16]).

\section{New observables for better sensitivity to breakup locations}

Having identified near-target breakup, an even finer distinction must be made which is of relevance to fusion suppression - whether breakup of the projectile-like nucleus occurs on the incoming trajectory or when receding from the target. In previous studies $[13,14,16]$, it has been assumed that of events with high relative energy, half arise from breakup on the incoming trajectory and half from the outgoing trajectory. This assumption is valid provided breakup (direct or following transfer) is instantaneous, since the probability of both direct excitation and transfer is expected to be symmetric around the turning point. However, if the unbound state of the nucleus (formed following direct excitation or nucleon transfer) has a lifetime, then it may continue on its trajectory before it breaks-up. Depending on the meanlife, the unbound state populated on the incoming trajectory may remain intact until it begins to recede from the target. It is therefore necessary [17] to have a more nuanced experimental observable than the fragment relative energy described in the previous section.

In the case of break-up close to the target, the fragments will be scattered to large angles due to their interaction with the target, resulting in a large angle $\theta_{12}$ between the two coincident fragments as shown in the inset of Fig. 
3 (a). On the other hand, if breakup occurs on the receding trajectory, far from the target, then the opening angle will on average be smaller. How different the opening angles will be in the two situations will depend on the target $Z$ and on the excitation energy of the nucleus that breaks-up. With heavy targets the strong Coulomb interaction will force the fragments to backward angles, which is likely to wash-out the distinction. Thus the distinction in opening angles for the incoming and outgoing breakup may be expected to be more visible for lighter targets. The distinction is clearly observed [17] in the reaction of ${ }^{7} \mathrm{Li}$ with ${ }^{58} \mathrm{Ni}$ by plotting the measured $\theta_{12}$ against the angle $\beta$ between the direction of the unbroken ${ }^{8} \mathrm{Be}$ and the deduced breakup axis, as shown in Fig. 3 (a). The data lie mainly in two distinct bands - one shows small $\theta_{12}$ for all values of $\beta$ with a tight correlation; the other has much larger $\theta_{12}$ with a larger spread for a given $\beta$. That such bands emerge from the fairly featureless (except for the peak at $92 \mathrm{keV}) E_{\text {rel }}$ spectrum shown in Fig. 2 is very interesting, and point to a common physical origin for events in each band. The superimposed lines in Fig. 3(a) show the expectations for asymptotic breakup of the ground state of ${ }^{8} \mathrm{Be}$ (dashed line) and $2^{+}$state of ${ }^{8} \mathrm{Be}$ (full line); as expected, there is a one-to-one correlation between $\beta$ and $\theta_{12}$, as effects of the Coulomb field of the target are negligible for asymptotic breakup. We see that the breakup of the ground state of ${ }^{8} \mathrm{Be}$ (that can be unambiguously identified from the $E_{r e l}$ value of $92 \mathrm{keV}$ ) follows the expectations beautifully. Clearly this state breaks up far from the target, consistent with its mean-life of $10^{-16} \mathrm{~s}$.

The other events, which include breakup from the $2^{+}$state of ${ }^{8} \mathrm{Be}$ with mean-life $0.4 \mathrm{zs}$, are not fully consistent with expectations of breakup far from the target (full line). As discussed earlier, large values of $\theta_{12}$ (strongly correlated with $\left.E_{r e l}\right)$ are indicative of breakup close to the target. Classical calculations that include the Coulomb effects due to proximity of the fragments to the target were done using the code PLATYPUS [15] to quantify the deviations from the asymptotic expectations. In order to illustrate the sensitivity of $\theta_{12}$ to the location of breakup, calculations were performed by varying the location of breakup with respect to the distance of closest approach. Representative calculations for a fixed excitation energy of ${ }^{8} \mathrm{Be}$ and fixed angular momentum are shown in Fig. 3(b) by lines that are superimposed on the experimental data. Each line corresponds to breakup at the indicated distance $\Delta r$ from the turning point on the outgoing trajectory. Variation in excitation energy and angular momentum will lead to some smearing around the lines shown. Calculations for breakup on the incoming trajectory (negative $\Delta r$ ) produce even larger $\theta_{12}$, which the current experimental arrangement was not sensitive to. However, the data and 

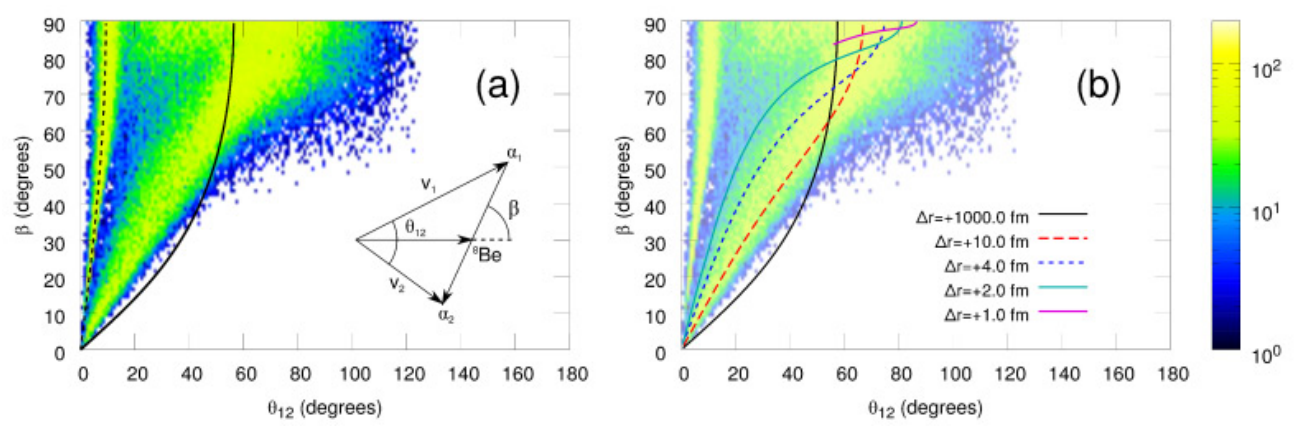

Figure 3: (Color online) The angle $\beta$ between the unbroken ${ }^{8} \mathrm{Be}$ and one of the breakup fragments is plotted against the angle between the two coincident fragments $\theta_{12}$. The angles $\beta$ and $\theta_{12}$ are illustrated in the inset in panel (a). The data for breakup of ${ }^{8} \mathrm{Be}$ formed in collisions of ${ }^{7} \mathrm{Li}$ with ${ }^{58} \mathrm{Ni}$ are shown in in both panels. Results of classical calculations are shown by the lines. Calculations in panel (a) show the expectations for asymptotic breakup of the ground state of ${ }^{8} \mathrm{Be}$ (dashed line) and the $2^{+}$state of ${ }^{8} \mathrm{Be}$ (full line). Calculations presented in panel (b) show the effect of changing the breakup position with respect to the target; a $\Delta r$ value of $1000 \mathrm{fm}$ corresponds to asymptotic breakup, and decreasing values of $\Delta r$ represent reducing distance from the turning point.

the simulations do illustrate the fact that the $\theta_{12}$ vs. $\beta$ plots are sensitive to breakup location [17], and thus can, in principle, be used to make a distinction between breakup on the incoming and outgoing trajectories.

\section{Summary}

Recent studies to understand the breakup of weakly bound light nuclei and its relationship to suppression of complete fusion have led to many new insights. It has been shown that direct breakup is not the only mode, and that breakup following nucleon transfer is extremely significant if transfer populates nuclei that are weakly bound. A more nuanced understanding of breakup locations can be obtained from measurements with detectors that cover a wide angular range. Comparison with classical simulations show that the mean-lives of the unbound states will play a role in determining the location of breakup. Experimental variables have been identified that allow us to probe this aspect and sensitivity to even sub-zeptosecond meanlife can be expected. These results provide insights into breakup dynamics near the barrier, and should help advance models to understand breakup and its effect on fusion. 


\section{Acknowledgments}

The authors acknowledge operations support for the ANU Heavy Ion Accelerator Facility from NCRIS. Financial support from ARC grants DP130101569, DE140100784, and FL110100098 is acknowledged.

\section{References}

[1] L.F. Canto et al., Phys. Rep. 596 (2015) 1, and references therein.

[2] M. Dasgupta et al., Phys. Rev. Lett 82 (1999) 1395.

[3] V. Tripathi et al., Phys. Rev. Lett. 88 (2002) 172701.

[4] Y.W. Wu et al., Phys. Rev. C 68 (2003) 044605.

[5] M. Dasgupta et al., Phys. Rev. C 70 (2004) 024606.

[6] A. Mukherjee et al., Phys. Lett. B 636 (2006) 91.

[7] P.K. Rath et al., Phys. Rev. C 79 (2009) 051601.

[8] L.R. Gasques et al., Phys. Rev. C 79 (2009) 034605.

[9] M. Dasgupta et al., Phys. Rev. C 81 (2010) 024608.

[10] N. Keeley et al., Prog. Part. Nucl. Phys. 59 (2007) 579.

[11] K.O. Pfeiffer et al., Nucl. Phys. A 206 (1973) 545.

[12] A. Shrivastava et al., Phys. Lett. B 633 (2006) 463.

[13] D.H. Luong et al., Phys. Lett. B 695 (2011) 105.

[14] D.H. Luong et al., Phys. Rev. C 88 (2013) 034609.

[15] A. Diaz-Torres et al., Phys. Rev. Lett. 98 (2007) 152701.

[16] R. Rafiei et al., Phys. Rev. C 81 (2010) 024601.

[17] E.C. Simpson et al., submitted to Phys. Rev. C (2015). 